\title{
Incidences and variations of hospital acquired venous thromboembolism in Australian hospitals: a population-based study
}

\author{
Hassan Assareh ${ }^{1,2^{*}}$, Jack Chen ${ }^{2}$, Lixin Ou², Ken Hillman ${ }^{2}$ and Arthas Flabouris ${ }^{3}$
}

\begin{abstract}
Background: Data on hospital-acquired venous thromboembolism (HA-VTE) incidence, case fatality rate and variation amongst patient groups and health providers is lacking. We aim to explore HA-VTE incidences, associated mortality, trends and variations across all acute hospitals in New South Wales (NSW)-Australia.

Methods: A population-based study using all admitted patients (aged 18-90 with a length of stay of at least two days and not transferred to another acute care facility) in 104 NSW acute public and private hospitals during 2002-2009. Poisson mixed models were used to derive adjusted rate ratios (IRR) in presence of patient and hospital characteristics.

Results: Amongst, 3,331,677 patients, the incidence of HA-VTE was 11.45 per 1000 patients and one in ten who developed HA-VTE died in hospital. HA-VTE incidence, initially rose, but subsequently declined, whereas case fatality rate consistently declined by $22 \%$ over the study period. Surgical patients were $128 \%$ (IRR $=2.28,95 \% \mathrm{Cl}: 2.19-2.38$ ) more likely to develop HA-VTE, but had similar case fatality rates compared to medical patients. Private hospitals, in comparison to public hospitals had a higher incidence of HA-VTE (IRR $=1.76 ; 95 \% \mathrm{Cl}$ : 1.42-2.18) for medical patients. However, they had a similar incidence (IRR $=0.91 ; 95 \% \mathrm{Cl}$ : 0.75-1.11), but a lower mortality (IRR $=0.59 ; 95 \% \mathrm{Cl}$ : 0.47-0.75) amongst surgical patients. Smaller public hospitals had a lower HA-VTE incidence rate compared to larger hospitals $(I R R<0.68)$ but a higher case fatality rate $(I R R>1.71)$. Hospitals with a lower reported HA-VTE incidence tended to have a higher HA-VTE case fatality rate.

Conclusion: Despite the decline in HA-VTE incidence and case fatality, there were large variations in incidents between medical and surgical patients, public and private hospitals, and different hospital groups. The causes of such differences warrant further investigation and may provide potential for targeted interventions and quality improvement initiatives.
\end{abstract}

Keywords: Hospital acquired complication, Patient safety, Quality improvement, Venous thromboembolism

\section{Background}

Venous thromboembolism (VTE), comprising both deep venous thrombosis (DVT) and pulmonary embolism $(\mathrm{PE})$ are potentially preventable and treatable medical conditions that can contribute to patient morbidity and

\footnotetext{
* Correspondence: hassan.assareh@health.nsw.gov.au

${ }^{1}$ Epidemiology and Health Analytics, Western Sydney Local Health Districts, Gungurra Building 68, Cumberland Hospital, 5 Fleet Street, North Parramatta 2151, NSW, Australia

${ }^{2}$ Simpson Centre for Health Services Research-South Western Sydney Clinical School Faculty of Medicine, University of New South Wales, and Ingham Institute, Sydney, Australia

Full list of author information is available at the end of the article
}

mortality [1, 2]. VTE accounts for almost $10 \%$ of all hospital deaths [3], and over half of VTE incidents are hospital acquire (HA-VTE) [4-6]. Appropriate intervention (e.g. pharmacological and mechanical prophylaxis), can significantly reduce the incidence of VTE by $70 \%$ for both medical and surgical patients [7-9]. Evidence-based VTE prevention and treatment guidelines $[1,10,11]$ have been developed so as to reduce VTE occurrence and have been adopted for both accreditation and benchmarking $[12,13]$.

In the U.S the incidence, and fatality rates, of HA-VTE following surgery have decreased by 50 and $30 \%$ 
respectively [14-16]. Effective implementation of VTE prevention and treatment protocols may have contributed to the decreased rates in the U.S. hospitals [17]. Substantial variation in trends and rates amongst similar hospitals [14], after adjustment for case mix and surgery types $[18,19]$, may reflect variation in compliance with VTE prevention strategies and the potential for further improvement [20]. In contrast, there has been a $30 \%$ increase in post-operative VTE incidents in Australian hospitals [21].

There have been few studies of the HA-VTE incidence and subsequnt mortality in Australia [21-24]. In this study, we adopted a validated measure of HA-VTE to explore trends in the rates of HA-VTE, and associated mortality amongst admitted patients to all acute public and private hospitals in New South Wales (NSW), Australia between 2002 and 2009. Patients' and hospitals' contributing factors to the HA-VTE trends and variations were also examined.

\section{Methods}

\section{Data source and study population}

We used records from the NSW Admitted Patient Data Collection (APDC) database. It includes all admitted patients in NSW public and private, acute, sub-acute and non-acute facilities, and is used for health services planning, funding and research. Reporting to APDC is mandatory at all hospitals. The APDC includes information on patient demographics, medical conditions and procedures, hospital characteristics, and separations (discharges, transfers and deaths) from all public and private healthcare facilities in NSW. Each episode of care in the APDC is assigned with up to 55 codes for morbidities (principal diagnosis and comorbidities) based on the International Statistical Classification of Diseases and Related Health Problems, Tenth Revision, Australian Modification (ICD-10-AM) 4th edition [25]. The codes are assigned by trained and qualified clinical coders based on clinical notes and according to standards [26]. The quality of data was found reasonable, but varied across data elements and hospitals [27-29].

In NSW public hospitals accommodated $62 \%$ of all patients' admissions, with a greater proportion of overnight stays (70 \%), but a similar same-day stays (51\%) compared to private hospitals. Over $80 \%$ of funding for public and private hospitals were provided by government and health insurers respectively [30]. All admissions, between 1st January 2002 to 31st December 2009, from 104 of 497 NSW acute public and private hospitals $(11,408,808$, admissions; $71 \%)$ were included. We excluded community, non-acute, psychiatric and rehabilitation facilities, nursing home and hospices, and the two children's hospitals.

Included were patients who had a hospital length of stay (LOS) of at least two days, aged between 18 and
90 years (inclusive), and were not transferred to another acute care facility $(4,089,144$ episodes $(35.8 \%))$.

\section{HA-VTE identification and covariates}

Based on ICD-10-AM, we considered the codes I26 (.0) and I26.9 for PE and I80.1, I80.2, I80.3, I80.8, I80.9, I82.8, and I82.9 for DVT cases - a total of nine VTE diagnosis codes. VTE codes were chosen according to well-established VTE related measures within a quality and safety context $[12,31,32]$ and existing published coding procedures $[19,22]$. Cases with no VTE code as the principal diagnosis were identified as patients at risk and those who had at least one VTE code in secondary diagnoses were identified as HA-VTE cases. It resulted in exclusion of patients with VTE as principal diagnoses from both numerator and denominator. Obstetric patients, identified by major diagnosis category using Australian Refined Diagnosis Related Groups (AR-DRG) [25], and those surgical patients who only had a procedure for interruption of vena cava (ICD codes: 348000 , 3533000 and 3533001) were excluded from the study population as suggested within a patient safety context in Australia [32] and elsewhere [31,33]. The discharge status (alive or deceased) was used to derive the case fatality associated with a HA-VTE diagnosis. HA-VTE cases and related deaths were respectively presented as incidence rate (per 1000 admissions) and case fatility.

For all admissions, two sets of patient- and hospitalrelated covariates were considered. Patient demographic variables included age, gender, country of birth, marital status, patient socio-economic status, principal diagnostic disease groups (the ten most common groups based on Elixhauser comorbidities [34]), and length of stay within the study population. We utilised a postcode-level advantage and disadvantage index of Socio-Economic Indices for Areas (SEIFA) with the lower values indicating more disadvantaged areas [35]. SEIFA scores were categorised into four classes (1st quartile $=$ most disadvantaged areas and 4th quartile $=$ most advantaged areas). The disease groups were identified using principal diagnostic codes (ICD-10-AM) at admissions through the methodology developed by Quan et al. [34]. Admissions with any surgical codes recored as the principal procedure were categorised as surgical admissions and those with no procedural data were categorised as medical admissions. Using relevant procedure codes from ICD-10-AM (Additional file 1), we defined six major surgical procedures including coronaryartery bypass graft (CABG), abdominal aortic aneurysm (AAA) repair, total hip replacement, total knee replacement, cholecystectomy, and other surgical procedures.

Hospital covariates included the local health district (metropolitan, rural and regional NSW), the hospital type (public and private), and associated peer groups. Public hospital peer groups included "A1": principal 
referral group, usually teaching hospitals; “A3”: ungrouped acute; "B": major metropolitan and non-metropolitan; "C1": district group 1; and, "C2": district group 2. Private hospital peer groups included "21": major; and "22": district group hospitals. Hospital peer groups contained similar type and sized hospitals, ranging from those treating more than 25,000 acute case-mix weighted separations per annum in principal referral groups through to treating 2000+ (but less than 5000) acute case-mix weighted separations per annum in district group [36].

\section{Statistical analysis}

We employed Poisson mixed models to evaluate adjusted incidence and case fatality rates and rate ratios for both study outcomes after including all patients and hospital-related characteristics. A random intercept term was utilised to incorporate any clustering effect at hospital-level. Stratified models were constructed to derive estimates for specific admission types (medical and surgical) and hospital peer groups (public and private) separately. To investigate the temporal pattern of the outcomes, calendar years were entered into the model as indicator variables, with 2002 as the reference year. A model with the year as a continuous variable was also examined for linear trends. We derived specific trends for hospital types, admission type, peer group and surgery type using separate models. Adjusted rates for specific years were derived by multiplying yearly-adjusted rate ratios to the crude rates observed in the reference year.

We did not include either the Elixhauser or the Charlson Index comorbidities in the models due to an approximately unexpected $50 \%$ drop (see Additional file 2) observed in the indices among our study population in recent years [21], and also reported geographical variations and biases in the coding that may lead to misleading results [37, 38]. For example, it was reported that there is an increased under-recording of diabetes conditions in APDC datasets between 2008 and 2012 [39].

To study the variation of outcomes across hospitals within each hospital group, hospital-specific random intercept components were extracted from stratified Poisson mixed models constructed for each hospital group, then ranked and categorised into five classes at $20 \%$ incremental quintiles. To obtain adjusted differences between those with the highest and those with the lowest HA-VTE and HA-VTE case fatality rates, the adjusted classes were entered into a Poisson model including patient characteristics covariates. We then used Pearson correlation to assess the association of hospital performances between HA-VTE and post HA-VTE deaths, based on the hospital-specific random intercepts. All analyses were performed in $\mathrm{R}$ package version 3.0.0 [40] and Stata $^{\mathrm{TM}} 11.0$ [41].

\section{Result}

The distributions of study outcomes by patient and hospital characteristics

Of the 3,331,677 admissions during 2002-2009 with a median LOS of five days ( $1^{\text {st }}$ and $3^{\text {rd }}$ quartiles: 3 , 9 days), 38,161 patients developed a HA-VTE, resulting in an incidence rate of 11.4 per 1000 patients (median LOS $=12$, quartiles: 7-22 days; Table 1). Among them, 3716 (9.7 \%) died in hospitals (median $\operatorname{LOS}=13$, quartiles: 7-25 days). Patients who underwent surgery (elective or non-elective, $83 \%$ of all included patients) accounted for $93.3 \%$ of HA-VTE cases and were 2.3 times more likely to develop HA-VTE compared to medical patients, but had a similar mortality rate. Compared to females, male patients were less likely to develop a HA-VTE (IRR $=0.93)$ but more likely to die $(I R R=1.25)$. HA-VTE incidence and associated case fatality were higher among elderly. Patients who stayed longer in hospitals had higher HA-VTE incidences but lower fatalities.

Patients who were born in Europe (except the UK), Asia and North Africa experienced a lower risk of postoperative HA-VTE but a similar risk of death. Higher socio-economic status (quartiles of SEIFA) was associated with a higher risk of post HA-VTE death, in particular for surgical patients. Surgical and medical patients admitted with pulmonary circulation or coagulopathy disorders had the highest HA-VTE incidences, followed by cancer patients who had the highest fatality rate. Patients who underwent total knee replacement, AAA repair and total hip replacement surgeries had a higher risk of HA-VTE. However, post HA-VTE mortality was lower amongst orthopaedic surgical patients, compared to other surgical patients (see Additional file 3).

For medical patients, HA-VTE incidence was significantly higher in private hospitals compared to public hospitals $(I R R=1.76)$, whereas for surgical patients it was similar (see Additional files 3 and 4). The risk of death in private hospitals was almost half of the risk in public hospitals, in particular for surgical patients.

Among public hospitals, patients from principal referral hospitals were more likely to acquire VTE during hospitalisation in comparison to patients from smaller hospitals. However, patients admitted to smaller hospitals, were at a higher risk of death. This pattern was almost consistent across medical and surgical patients (see Additional files 3 and 4). No differences in outcomes were observed between private hospital peer groups.

\section{The trends of HA-VTE incidence rate and HA-VTE case fatality over the study period}

HA-VTE incidence rate increased by $14 \%$ between 2002 and 2004 reaching an adjusted incidence rate of 12.4 per 1000 patients, and then declined during the rest of the study period (Fig. 1). The increase in the HA-VTE incidence rate between 2002 and 2004 occurred in surgical but not 
Table 1 Study population, incidence rates and adjusted rate ratios of patients who developed HA-VTE and associated case fatality

\begin{tabular}{|c|c|c|c|c|c|c|c|c|c|c|c|c|c|c|}
\hline \multirow[t]{2}{*}{ Characteristics } & \multicolumn{2}{|c|}{ All Patients } & \multicolumn{6}{|l|}{ HA-VTE } & \multicolumn{6}{|c|}{ HA-VTE case fatality } \\
\hline & $\mathrm{N}$ & $(\%)$ & $n$ & $(\%)$ & IR & IRR & $(95 \% \mathrm{Cl})$ & & $n$ & $(\%)$ & $\%$ & IRR & $(95 \% \mathrm{Cl})$ & \\
\hline \multicolumn{15}{|l|}{ Admission type $^{a}$} \\
\hline Medical & 573,042 & (17.20 \%) & 2,565 & $(6.72 \%)$ & 4.5 & 1.00 & & & 245 & (6.59 \%) & $9.6 \%$ & 1.00 & & \\
\hline Surgical & $2,758,635$ & (82.80 \%) & 35,596 & (93.28 \%) & 12.9 & 2.28 & $(2.19-2.38)$ & d & 3,471 & (93.41\%) & $9.8 \%$ & 1.05 & $(0.91-1.20)$ & \\
\hline \multicolumn{15}{|l|}{ Sex } \\
\hline Female & $1,693,109$ & (50.82 \%) & 19,805 & (51.90 \%) & 11.7 & 1.00 & & & 1,730 & (46.56 \%) & $8.7 \%$ & 1.00 & & \\
\hline Male & $1,638,568$ & (49.18 \%) & 18,356 & (48.10 \%) & 11.2 & 0.93 & $(0.91-0.95)$ & d & 1,986 & (53.44 \%) & $10.8 \%$ & 1.25 & $(1.17-1.33)$ & d \\
\hline \multicolumn{15}{|l|}{ Age } \\
\hline$>=75$ years $\&<90$ & $1,074,206$ & (32.24 \%) & 15,350 & (40.22 \%) & 14.3 & 1.00 & & & 1,832 & (49.30 \%) & $11.9 \%$ & 1.00 & & \\
\hline$>=18$ years $\&<35$ years & 396,444 & (11.90 \%) & 1,742 & $(4.56 \%)$ & 4.4 & 0.53 & $(0.50-0.56)$ & d & 55 & $(1.48 \%)$ & $3.2 \%$ & 0.23 & $(0.17-0.30)$ & d \\
\hline$>=35$ years $\&<55$ years & 707,274 & (21.23 \%) & 5,612 & (14.71\%) & 7.9 & 0.83 & $(0.81-0.86)$ & d & 354 & (9.53 \%) & $6.3 \%$ & 0.47 & $(0.41-0.52)$ & ${ }^{d}$ \\
\hline$>=55$ years $\&<75$ years & $1,153,753$ & (34.63 \%) & 15,457 & $(40.50 \%)$ & 13.4 & 1.13 & $(1.10-1.15)$ & d & 1,475 & (39.69 \%) & $9.5 \%$ & 0.77 & $(0.72-0.83)$ & d \\
\hline \multicolumn{15}{|l|}{ Marital status } \\
\hline Married & $1,803,208$ & (54.12 \%) & 20,814 & (54.54 \%) & 11.5 & 1.00 & & & 2,089 & (56.22 \%) & $10.0 \%$ & 1.00 & & \\
\hline Single & $1,372,496$ & (41.20 \%) & 15,730 & (41.22 \%) & 11.5 & 0.90 & $(0.89-0.92)$ & $d$ & 1,447 & (38.94 \%) & $9.2 \%$ & 0.91 & $(0.85-0.98)$ & ${ }^{c}$ \\
\hline Unknown & 155,973 & $(4.68 \%)$ & 1,617 & $(4.24 \%)$ & 10.4 & 0.83 & $(0.79-0.88)$ & d & 180 & $(4.84 \%)$ & $11.1 \%$ & 1.12 & $(0.96-1.31)$ & \\
\hline \multicolumn{15}{|l|}{ Country of birth } \\
\hline Australia and New Zealand & $2,411,315$ & (72.38 \%) & 26,794 & (70.21 \%) & 11.1 & 1.00 & & & 2,575 & (69.29 \%) & $9.6 \%$ & 1.00 & & \\
\hline UK, US \& Canada & 223,710 & $(6.71 \%)$ & 2,873 & $(7.53 \%)$ & 12.8 & 1.01 & $(0.97-1.05)$ & & 271 & $(7.29 \%)$ & $9.4 \%$ & 0.90 & $(0.79-1.02)$ & \\
\hline Non-English Europe & 317,973 & $(9.54 \%)$ & 4,546 & (11.91\%) & 14.3 & 0.98 & $(0.95-1.02)$ & & 493 & $(13.27 \%)$ & $10.8 \%$ & 0.98 & $(0.88-1.08)$ & \\
\hline North Africa & 61,595 & $(1.85 \%)$ & 487 & $(1.28 \%)$ & 7.9 & 0.67 & $(0.61-0.73)$ & d & 47 & $(1.26 \%)$ & $9.7 \%$ & 1.09 & $(0.82-1.46)$ & \\
\hline Asia & 101,485 & $(3.05 \%)$ & 996 & $(2.61 \%)$ & 9.8 & 0.76 & $(0.71-0.81)$ & d & 98 & $(2.64 \%)$ & $9.8 \%$ & 1.11 & $(0.90-1.36)$ & \\
\hline Others & 215,599 & $(6.47 \%)$ & 2,465 & $(6.46 \%)$ & 11.4 & 0.94 & $(0.90-0.98)$ & d & 232 & (6.24 \%) & $9.4 \%$ & 1.01 & $(0.88-1.16)$ & \\
\hline \multicolumn{15}{|l|}{ Quartiles of SEIFA } \\
\hline 1st quartile (most disadvantaged) & 913,712 & $(27.42 \%)$ & 9,482 & (24.85\%) & 10.4 & 1.00 & & & 939 & (25.27 \%) & $9.9 \%$ & 1.00 & & \\
\hline 2nd quartile (disadvantaged) & 862,580 & $(25.89 \%)$ & 8,843 & $(23.17 \%)$ & 10.3 & 1.00 & $(0.97-1.03)$ & & 900 & (24.22 \%) & $10.2 \%$ & 1.05 & $(0.95-1.16)$ & \\
\hline 3rd quartile (advantaged) & 827,343 & $(24.83 \%)$ & 9,701 & (25.42 \%) & 11.7 & 0.98 & $(0.95-1.01)$ & & 1,011 & (27.21 \%) & $10.4 \%$ & 1.12 & $(1.01-1.23)$ & c \\
\hline 4th quartile (most advantaged) & 728,042 & $(21.85 \%)$ & 10,135 & $(26.56 \%)$ & 13.9 & 0.99 & $(0.95-1.03)$ & & 866 & (23.30 \%) & $8.5 \%$ & 1.13 & $(1-1.27)$ & c \\
\hline \multicolumn{15}{|l|}{ Length of stay } \\
\hline 2-4 days & $1,495,477$ & $(44.89 \%)$ & 4,803 & $(12.59 \%)$ & 3.2 & 1.00 & & & 567 & $(15.26 \%)$ & $11.8 \%$ & 1.00 & & \\
\hline 4-9 days & $1,056,167$ & (31.70\%) & 10,724 & (28.10 \%) & 10.2 & 2.78 & $(2.69-2.88)$ & d & 816 & (21.96\%) & $7.6 \%$ & 0.69 & $(0.62-0.76)$ & d \\
\hline Over 10 days & 780,033 & $(23.41 \%)$ & 22,634 & (59.31\%) & 29.0 & 7.91 & $(7.65-8.17)$ & d & 2,333 & $(62.78 \%)$ & $10.3 \%$ & 0.82 & $(0.74-0.90)$ & 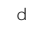 \\
\hline \multicolumn{15}{|l|}{ Major principal diagnostic diseases ${ }^{b}$} \\
\hline Cardiac arrhythmias & 74,686 & $(2.24 \%)$ & 657 & $(1.72 \%)$ & 8.8 & - & & & 22 & $(0.59 \%)$ & $3.3 \%$ & - & & \\
\hline Chronic pulmonary disease & 129,030 & $(3.87 \%)$ & 853 & $(2.24 \%)$ & 6.6 & - & & & 92 & $(2.48 \%)$ & $10.8 \%$ & - & & \\
\hline Coagulopathy & 5,455 & $(0.16 \%)$ & 211 & $(0.55 \%)$ & 38.7 & - & & & 15 & $(0.40 \%)$ & $7.1 \%$ & - & & \\
\hline Congestive heart failure & 71,705 & $(2.15 \%)$ & 971 & $(2.54 \%)$ & 13.5 & - & & & 130 & (3.50 \%) & $13.4 \%$ & - & & \\
\hline Diabetes with chronic complication & 41,956 & $(1.26 \%)$ & 464 & $(1.22 \%)$ & 11.1 & - & & & 58 & $(1.56 \%)$ & $12.5 \%$ & - & & \\
\hline Malignancies & 206,407 & $(6.20 \%)$ & 3,796 & $(9.95 \%)$ & 18.4 & - & & & 860 & (23.14 \%) & $22.7 \%$ & - & & \\
\hline Metastatic solid tumour & 49,332 & $(1.48 \%)$ & 1,427 & $(3.74 \%)$ & 28.9 & - & & & 368 & (9.90 \%) & $25.8 \%$ & - & & \\
\hline Peripheral vascular disease & 26,225 & $(0.79 \%)$ & 425 & $(1.11 \%)$ & 16.2 & - & & & 61 & $(1.64 \%)$ & $14.4 \%$ & - & & \\
\hline Pulmonary circulation disorders & 1,520 & $(0.05 \%)$ & 73 & $(0.19 \%)$ & 48.0 & - & & & 10 & $(0.27 \%)$ & $13.7 \%$ & - & & \\
\hline Renal disease & 8,736 & $(0.26 \%)$ & 103 & $(0.27 \%)$ & 11.8 & - & & & 14 & (0.38 \%) & $13.6 \%$ & - & & \\
\hline Rheumatic disease & 9,811 & $(0.29 \%)$ & 176 & $(0.46 \%)$ & 17.9 & - & & & 9 & $(0.24 \%)$ & $5.1 \%$ & - & & \\
\hline
\end{tabular}


Table 1 Study population, incidence rates and adjusted rate ratios of patients who developed HA-VTE and associated case fatality (Continued)

\begin{tabular}{|c|c|c|c|c|c|c|c|c|c|c|c|c|c|c|}
\hline \multicolumn{15}{|l|}{ Hospital type } \\
\hline Public & $2,704,301$ & (81.17\%) & 31,160 & (81.65 \%) & 11.5 & 1.00 & & & 3,362 & (90.47\%) & $10.8 \%$ & 1.00 & & \\
\hline Private & 627,376 & $(18.83 \%)$ & 7,001 & (18.35\%) & 11.2 & 1.03 & $(0.85-1.25)$ & & 354 & (9.53 \%) & $5.1 \%$ & 0.55 & $(0.43-0.71)$ & $d$ \\
\hline \multicolumn{15}{|l|}{ Peer hospital groups-Public } \\
\hline Principal referral (A1) & $1,315,038$ & $(48.63 \%)$ & 19,134 & (61.41\%) & 14.6 & 1.00 & & & 2,069 & $(61.54 \%)$ & $10.8 \%$ & 1.00 & & \\
\hline Ungrouped acute (A3) & 73,569 & $(2.72 \%)$ & 765 & $(2.46 \%)$ & 10.4 & 0.67 & $(0.46-0.95)$ & c & 132 & (3.93 \%) & $17.3 \%$ & 2.71 & $(1.48-4.97)$ & d \\
\hline Major metro- \& non-metropolitan (B) & 839,827 & (31.06 \%) & 7,958 & (25.54 \%) & 9.5 & 0.73 & $(0.60-0.89)$ & $d$ & 817 & (24.30 \%) & $10.3 \%$ & 1.22 & $(0.91-1.64)$ & \\
\hline District group 1 (C1) & 245,005 & (9.06 \%) & 1,933 & $(6.20 \%)$ & 7.9 & 0.64 & $(0.51-0.81)$ & $d$ & 198 & (5.89 \%) & $10.2 \%$ & 1.75 & $(1.22-2.50)$ & d \\
\hline District group 2 (C2) & 230,862 & (8.54 \%) & 1,370 & $(4.40 \%)$ & 5.9 & 0.48 & $(0.39-0.60)$ & $d$ & 146 & $(4.34 \%)$ & $10.7 \%$ & 2.06 & $(1.44-2.95)$ & d \\
\hline \multicolumn{15}{|l|}{ Peer hospital groups-Private } \\
\hline Major $\mathrm{m}$ & 409,555 & $(65.28 \%)$ & 4,961 & (70.86 \%) & 12.1 & 1.00 & & & 213 & $(60.17 \%)$ & $4.3 \%$ & 1.00 & & \\
\hline District (22) & 217,821 & (34.72 \%) & 2,040 & (29.14 \%) & 9.4 & 1.03 & $(0.64-1.64)$ & & 141 & (39.83 \%) & $6.9 \%$ & 1.36 & $(0.79-2.34)$ & \\
\hline \multicolumn{15}{|l|}{ Local health district } \\
\hline Metropolitan & $2,300,073$ & (69.04 \%) & 29,779 & (78.04 \%) & 12.9 & 1.00 & & & 2,814 & (75.73 \%) & $9.4 \%$ & 1.00 & & \\
\hline Rural \& Regional NSW & $1,031,604$ & (30.96 \%) & 8,382 & (21.96\%) & 8.1 & 0.69 & $(0.59-0.81)$ & $d$ & 902 & (24.27\%) & $10.8 \%$ & 1.18 & $(0.95-1.45)$ & \\
\hline Total & $3,331,677$ & & 38,161 & & 11.4 & & & & 3,716 & & $9.7 \%$ & & & \\
\hline
\end{tabular}

111,493 (3.2 \%) cases were excluded due to missing items Incidence rates (IR) are crude and reported per 1000 patients

Incidence rate ratios (IRR) and related confident intervals (CI) were obtained using a Poisson mixed model and adjusted for patient and hospital characteristics aIRRs were not adjusted for length of stay due to highly unbalanced distributions for medical versus surgical admissions

${ }^{b}$ No RR is reported since this characteristic has not been included in the Poisson mixed model

'Significant at $5 \%$; ${ }^{d}$ significant at $1 \%$

medical patients in all hospitals (Fig. 2). However, in private hospitals, the incidence rate of HA-VTE peaked later, with greater increments of 44 and $40 \%$ in 2006 and 2007 for surgical and medical patients, respectively (Fig. 2 and Additional file 4). Higher variations were observed in stratified trends - by public versus private, types of patients, hospital peer groups and surgical types (see Additional file 5).

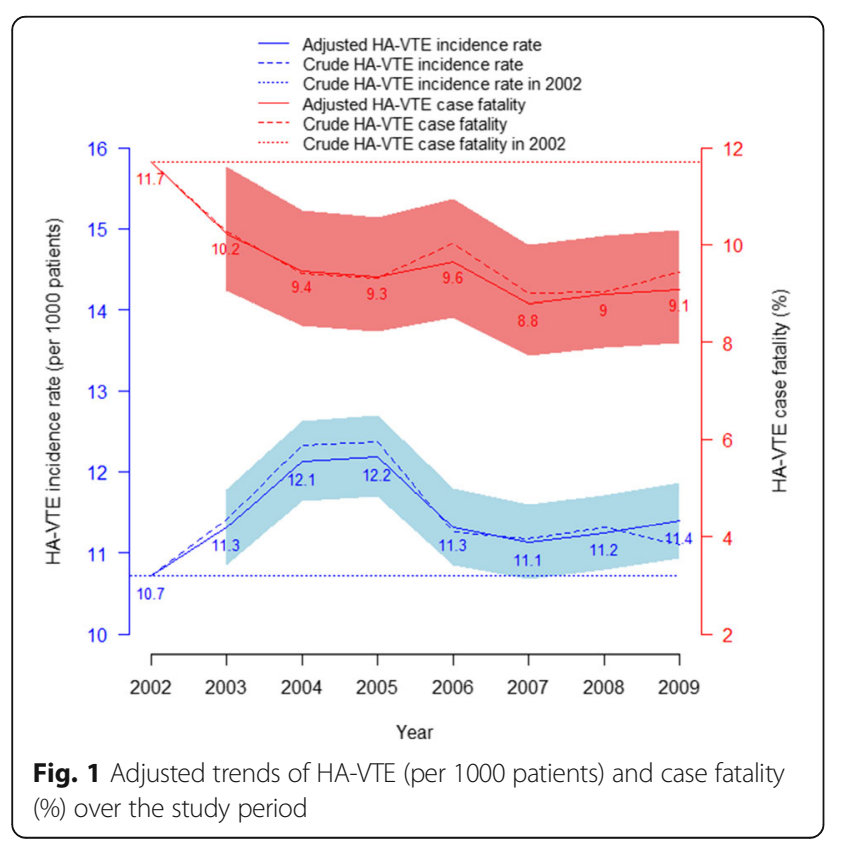

The adjusted HA-VTE case fatality rate significantly declined by $22 \%$ from 11.7 to $9.1 \%$ over the study period (Fig. 1). Stratified analysis illustrated in Fig. 3 showed similar declines between public (2009 vs 2002: IRR $=$ 0.74 ) and private hospitals (2009 vs 2002: $I R R=0.88$ ), surgical $(I R R=0.80)$ and medical patients $(I R R=0.59)$. Large principal referral and metropolitan hospitals

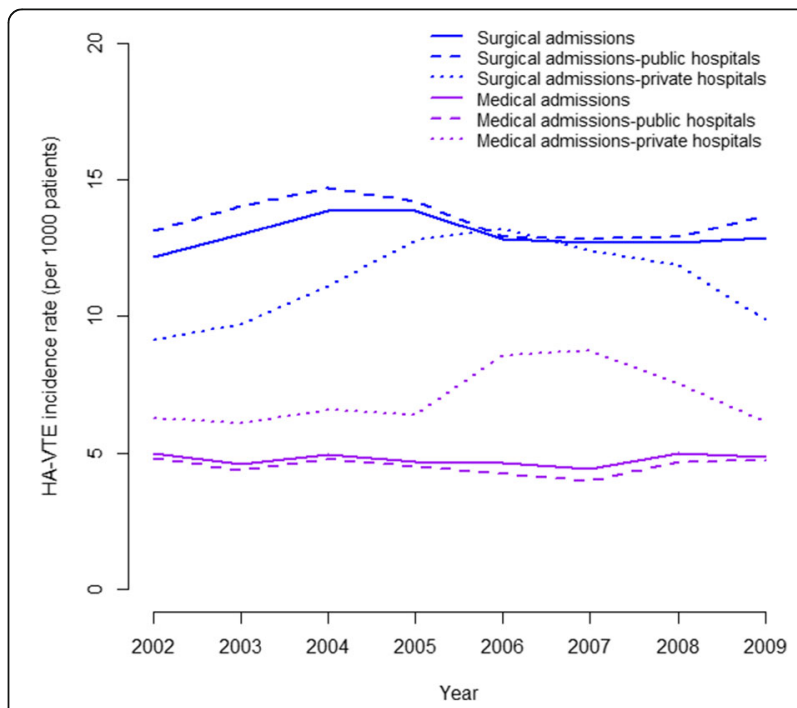

Fig. 2 Adjusted trend of HA-VTE stratified by admission and hospital type over the study period 


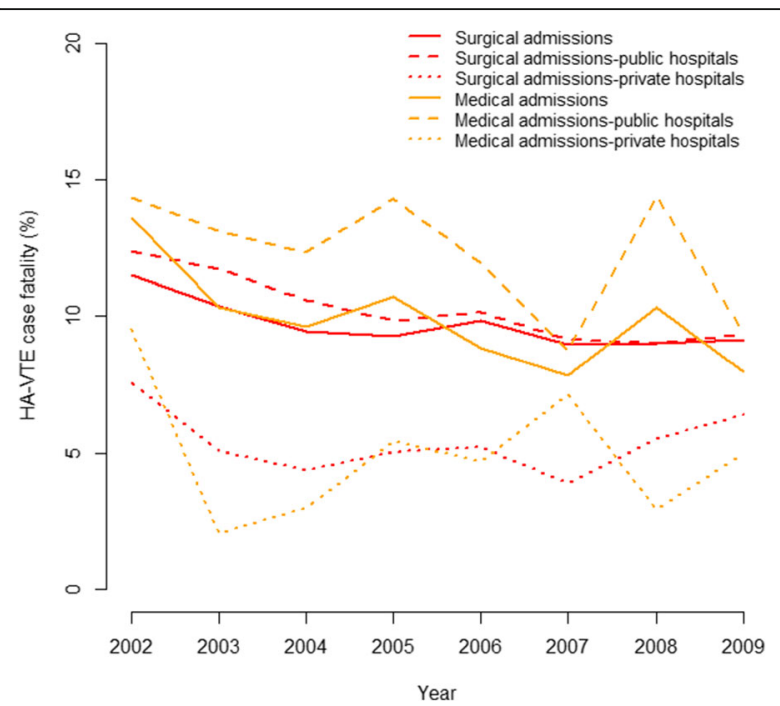

Fig. 3 Adjusted trend of post HA-VTE case fatality stratified by admission and hospital type over the study period

(coded as A1 and B) exhibited a significant decrease in the HA-VTE associated fatality rate over the study period (see Additional file 5).

\section{The variation of outcomes between and with hospital peer groups stratified by patients types (medical versus surgical)}

Table 2 shows that private hospitals exhibited a larger variation between those hospitals with the lowest, and those with the highest HA-VTE rate, and associated fatality rates within each peer groups compared to public hospitals peer groups. The major private hospital peer group showed the highest variability of HA-VTA between the top and bottom $20 \%$ percentile performers (IRR $=5.33)$, followed by district group 2 public hospitals (IRR $=2.94$ ) and district private hospital groups (IRR $=2.44)$. For HA-VTE case fatality, the major private hospital group had the largest variability (IRR $=6.23$ ), followed by the district private hospital group (IRR $=5.72)$ and then the district group 1 public hospitals (IRR $=3.77$ ). These within group variations were almost replicated for surgical patients, whereas for medical patients, the district group 1 public hospital showed the highest variability for HA-VTE rates (IRR $=4.32)$ and principal referral public hospital group had the highest variability for HA-VTE case fatality $(I R R=15.65)$. The significant negative correlations between individual hospital HA-VTE rates and HA-VTEs case fatality rates for private hospital peer groups implied that hospitals with the highest HA-VTE rate tended to have a lower rate of subsequent death. There were no such associations within other public hospitals peer groups.

\section{Discussion}

In this large cohort study of all NSW acute hospitals during 2002-2009, we found that over 1 in 100 patients developed HA-VTE. Of these, one in ten subsequently died during hospitalisation. The adjusted HA-VTE incidence peaked in 2004 and then declined afterwards, while the HA-VTE case fatality decreased by $22 \%$ over the study period. Risk of HA-VTE for surgical patients was double the risk for medical patients, but fatality rate was similar. Medical patients admitted at private hospitals experienced higher risks of HA-VTE; whereas surgical patients were at higher risk of subsequent death in public hospitals. There were significant variations in HA-VTE incidence and case fatality between, and within, hospital peer groups. Among public hospitals, principal referral hospitals had a higher HA-VTE incidence but lower associated deaths compared to the smaller hospitals. Whereas there was no difference between major and smaller private hospitals. Overall, between-hospital variation tended to be higher among private hospitals, particularly for major facilities. Smaller public hospitals often exhibited a larger variation in both rates.

The incidence of HA-VTE in NSW hospitals was almost five fold the rate (11.4 vs 2.4 per 1000 patients with VTE as secondary diagnoses) previously reported in Australia (Australian Institute of Health and Welfare (AIHW) 2010). Our rate among surgical patients was higher than those reported for patients who underwent elective surgery in NSW or U.S. hospitals (13 vs $2-4.5$ per 1000 patients) [12, 14, 21]. In contrast, our observed rate for medical patients was broadly consistent with those reported for U.S. hospitals (4.5 vs 2.5 to 5.1 per 1000 patients) [42, 43]. No comparable measures are available for overall HA-VTE case fatality in an Australian setting. For surgical patients, case fatality rate was similar to the previous report in NSW (9.8 \% vs. 7.9 and $8.3 \%)$ $[14,21]$; for medical patients, it was lower than the U.S hospitals' rate (9.6\% vs 15 to $16.5 \%$ ) [42, 44]. Differences in case identification introduced by varying ICD codes and systems, and inclusion of VTE incidents for all surgical patients in this study, compared to patients who just underwent elective surgery in other reports may have contributed to the discrepancies.

Patients with cancer, vascular and clotting disorders are at high risk of developing VTE [5, 44]. The high fatality rate found among cancer patients is consistent with previous studies reporting cancer as a major risk factor of developing PE and a high fatality rate [45]. Our finding that medical patients were less likely to develop VTE compared to surgical patients echoes the fact that surgery and in particular major procedures such as orthopaedic or AAA repair are known risk factors $[1,21,46]$. 
Table 2 Rates, adjusted rate ratios and association of outcomes between the best and worst performers within hospital peer groups

\begin{tabular}{|c|c|c|c|c|c|c|c|c|c|c|c|c|c|c|}
\hline \multirow[t]{2}{*}{ Hospital peer group } & \multirow{2}{*}{$\begin{array}{l}\text { Hospital } \\
n\end{array}$} & \multicolumn{5}{|l|}{ HA-VTE } & \multicolumn{5}{|c|}{ HA-VTE case fatality } & \multirow{2}{*}{\multicolumn{3}{|c|}{$\frac{\text { Correlation coefficient }}{(95 \% \mathrm{Cl})}$}} \\
\hline & & Lowest (IR) & Highest (IR) & IRR $(9$ & $95 \%(\mathrm{Cl})$ & & Lowest (\%) & Highest (\%) & IRR $(95$ & $\%(\mathrm{Cl})$ & & & & \\
\hline \multicolumn{15}{|l|}{ Public hospitals } \\
\hline Principal referral (A1) & 17 & 11.0 & 22.9 & 2.09 & $(2-2.19)$ & b & $7.7 \%$ & $13.8 \%$ & 1.93 & $(1.64-2.27)$ & b & -0.38 & $(-0.76-0.19)$ & \\
\hline $\begin{array}{l}\text { Major metro- \& } \\
\text { non-metropolitan (B) }\end{array}$ & 22 & 6.8 & 12.6 & 1.70 & $(1.58-1.84)$ & b & $6.9 \%$ & $15.0 \%$ & 2.13 & $(1.73-2.63)$ & b & -0.22 & $(-0.59-0.22)$ & \\
\hline District group 1 (C1) & 13 & 5.8 & 11.7 & 2.02 & $(1.77-2.31)$ & b & $5.9 \%$ & $20.5 \%$ & 3.77 & $(2.41-5.91)$ & $\mathrm{b}$ & -0.35 & $(-0.76-0.25)$ & \\
\hline District group 2 (C2) & 30 & 2.8 & 10.4 & 2.94 & $(2.39-3.62)$ & b & $4.7 \%$ & $14.7 \%$ & 3.47 & $(1.94-6.19)$ & $\mathrm{b}$ & 0.04 & $(-0.33-0.39)$ & \\
\hline \multicolumn{15}{|l|}{ Private hospitals } \\
\hline Major (21) & 10 & 4.0 & 24.7 & 5.33 & $(4.62-6.16)$ & b & $1.4 \%$ & $10.5 \%$ & 6.23 & $(4.25-9.13)$ & b & -0.63 & $(-0.9-0.01)$ & a \\
\hline District (22) & 12 & 6.0 & 14.3 & 2.44 & $(2.05-2.9)$ & b & $2.8 \%$ & $12.3 \%$ & 5.72 & $(3.1-10.58)$ & $\mathrm{b}$ & -0.82 & $(-0.95-0.46)$ & b \\
\hline \multicolumn{15}{|l|}{ Medical admissions } \\
\hline \multicolumn{15}{|l|}{ Public hospitals } \\
\hline Principal referral (A1) & 17 & 3.3 & 7.6 & 2.24 & $(1.75-2.87)$ & b & $1.3 \%$ & $18.3 \%$ & 15.65 & $(3.73-65.58)$ & b & -0.08 & $(-0.61-0.49)$ & \\
\hline $\begin{array}{l}\text { Major metro- \& } \\
\text { non-metropolitan (B) }\end{array}$ & 22 & 2.3 & 4.5 & 2.03 & $(1.59-2.58)$ & b & $0.0 \%$ & $20.1 \%$ & & & & 0.29 & $(-0.15-0.63)$ & \\
\hline District group 1 (C1) & 13 & 1.5 & 5.5 & 4.32 & $(2.72-6.86)$ & b & $4.0 \%$ & $20.0 \%$ & 5.81 & $(1.58-21.41)$ & b & -0.36 & $(-0.76-0.23)$ & \\
\hline District group 2 (C2) & 30 & 2.3 & 7.0 & 3.65 & $(2.64-5.06)$ & b & $0.0 \%$ & $27.8 \%$ & & & & -0.13 & $(-0.47-0.24)$ & \\
\hline \multicolumn{15}{|l|}{ Private hospitals } \\
\hline Major (21) & 10 & 5.1 & 13.6 & 2.52 & $(1.48-4.29)$ & b & $1.1 \%$ & $20.0 \%$ & & & & 0.32 & $(-0.39-0.79)$ & \\
\hline District (22) & 12 & 3.6 & 12.9 & 3.88 & $(2.55-5.92)$ & b & $4.6 \%$ & $16.7 \%$ & 2.57 & $(0.66-9.97)$ & & -0.67 & $(-0.9-0.16)$ & a \\
\hline \multicolumn{15}{|l|}{ Surgical admissions } \\
\hline \multicolumn{15}{|l|}{ Public hospitals } \\
\hline Principal referral (A1) & 17 & 11.8 & 25.0 & 2.12 & $(2.02-2.22)$ & b & $8.9 \%$ & $14.0 \%$ & 1.74 & $(1.52-1.99)$ & $\mathrm{b}$ & -0.38 & $(-0.76-0.19)$ & \\
\hline $\begin{array}{l}\text { Major metro- \& } \\
\text { non-metropolitan (B) }\end{array}$ & 22 & 7.9 & 14.3 & 1.74 & $(1.61-1.88)$ & b & $7.2 \%$ & $14.7 \%$ & 2.01 & $(1.62-2.49)$ & b & -0.21 & $(-0.58-0.23)$ & \\
\hline District group 1 (C1) & 13 & 7.2 & 13.7 & 1.88 & $(1.63-2.16)$ & b & $5.8 \%$ & $20.8 \%$ & 4.11 & $(2.5-6.75)$ & $\mathrm{b}$ & -0.30 & $(-0.73-0.3)$ & \\
\hline District group 2 (C2) & 30 & 2.9 & 14.7 & 3.75 & $(2.83-4.98)$ & b & $3.8 \%$ & $14.9 \%$ & 4.37 & $(2.06-9.26)$ & $\mathrm{b}$ & 0.05 & $(-0.32-0.4)$ & \\
\hline \multicolumn{15}{|l|}{ Private hospitals } \\
\hline Major (21) & 10 & 3.6 & 25.0 & 6.07 & $(5.2-7.09)$ & b & $1.7 \%$ & $11.2 \%$ & 5.88 & (3.77-9.18) & $\mathrm{b}$ & -0.36 & $(-0.81-0.35)$ & \\
\hline District (22) & 12 & 5.9 & 11.8 & 2.37 & $(1.99-2.81)$ & b & $2.2 \%$ & $11.3 \%$ & 6.88 & (3.24-14.62) & b & -0.72 & $(-0.92-0.25)$ & b \\
\hline
\end{tabular}

Incidence rates (IR) are crude and reported per 1000 patients

Incidence rate ratios (IRR) and related confident intervals $(\mathrm{Cl})$ were obtained using Poisson mixed models and adjusted for patient characteristics. Those hospitals with the lowest rate were set as the reference level

Ungrouped acute peer group within public hospitals was removed from analysis due to small number of hospitals within this group

${ }^{a}$ Significant at $5 \%$; ${ }^{b}$ significant at $1 \%$

The recording and reporting of post-operative VTE as a patient safety outcome may have contributed to halving of the HA-VTE rates amongst surgical patients in the U.S. $[12,15]$. No such a drop in Australia was evident $[21,23]$. However, the decreasing trajectory in HA-VTE incidents and in particular in associated mortality after adjusting for varied length of stay over time, coincided with the release and adherence with VTE prevention guidelines in Australia [2, 47].

Compliance with VTE prevention guidelines was reportedly suboptimal for hospitalised patients and medical patients were up to $50 \%$ less likely than surgical patients to receive adequate VTE prophylaxis [48-50]. Other studies have shown a smaller improvement in post VTE survival for medical patients [51,52] which was not evident in our study. Extension of the VTE prevention efforts to medical patients as recently targeted in Australia [11] and elsewhere [53] and routine measurement of associated indictors could reduce incidences and adverse outcome.

We found that the incidence of HA-VTE was lower for medical patients at public versus private hospitals; but public hospitals had higher subsequent case fatalities, in particular for surgical patients. Differences in compliance with VTE prevention guidelines can result in varying VTE measures across hospitals. Altered length of stay have been contributed to the differences in HA-VTE outcomes 
between two sectors [44, 54], however we here adjusted for length of stay across hospitals, offsetting the influence of shorter stay in private hospitals [30]. A difference in case mix may have contributed to discrepancies between the two sectors.

Our finding that NSW larger hospitals had excess HA-VTE incidents but lower fatalities is consistent with hospital-volume effect reported for HA-VTE [21] and mortality [55-57]. Undertaking patients with higher surgical complexity and multiple comorbidities as well as better diagnosis of high-risk but asymptomatic VTE in larger hospitals [58] may have contributed to elevated HA-VTE rates in major hospitals. On the other hand, underreporting, failure to diagnose or a mis-coding of HA-VTE may be more common amongst smaller hospitals [22, 37, 59]. Subsequently, majority of the identified VTE cases in smaller hospitals are those who developed $\mathrm{PE}$ and were more likely to die. A higher rate of diagnosis, and lower case fatality, may reflect a greater adherence to evidence-based treatment guidelines and less failure-torescue. Therefore, study of HA-VTE incidents alone may be misleading and more research is required to identify contributing factors to both the incidence rate and case fatality.

Our study has several implications. Expansion of VTE prevention programs to private hospitals and medical patients are required. The large variation of HA-VTE rates between and within different sectors and hospital peer groups suggests that there is room for improvement in the diagnosis, prevention and treatment of HA-VTE. Development of measures for hospital acquired VTE, and the continual monitoring and public reporting of the VTE incidence and mortality for benchmarking and quality improvement purposes at local, regional and national level should be considered $[2,60]$.

This study benefited from a large population-based cohort across all acute hospitals within the most populated region in Australia. We utilised two measures that were comparable to well-established VTE and case fatality indicators within the patient safety literature, which allowed us to differentiate prevention versus treatment of VTE as well as benchmarking internally and externally. However our study has multiple limitations. The rates of HA-VTE may be prone to two biases: a) overestimation due to possible inclusion of community base VTE cases that were recorded as secondary diagnoses; and b) underestimation due to neglecting VTE cases developed during hospitalisation but not diagnosed prior to discharge [44]. This suggests that bias may be reduced by the utilisation of newly adopted condition onset variables (whether or not morbidity was present on admission) in hospital administrative datasets [61] and data linkage in the follow-up period [62]. The accuracy of our results were limited to the quality of documentation and clinical coding practices [37], and thus can be improved via clinical chart review. Furthermore inclusion of broader confounding factors such as severity of patients such as Charlson and Elixhauser scores based on accurate morbidity data, body mass index and smoking can provide additional insight on the observed trends $[63,64]$.

\section{Conclusion}

There incidence and case fatality rate of HA-VTE in NSW hospital in-patients is decreasing. The significant variability for HA-VTE incidents and case fatality between medical and surgical patients within public and private sectors and between hospital peer groups suggests potential for further improvement in both prevention and treatment of HA-VTE. Routine measurement and reporting of both HA-VTE incidence and associated mortality can provide policy-makers, clinicians and researchers with opportunities to monitor and improve performance.

\section{Additional files}

Additional file 1: Procedure codes from ICD-10-AM for selected surgical procedures. Procedure codes from ICD-10-AM for selected surgical procedures. (DOCX $13.2 \mathrm{~kb}$ )

Additional file 2: Patients' age and severity over the study period. (DOCX $13.8 \mathrm{~kb}$ )

Additional file 3: Study population, incidence rates and adjusted rate ratios of surgical patients who developed HA-VTE and associated case fatality. (DOCX 29 kb)

Additional file 4: Study population, incidence rates and adjusted rate ratios of medical patients who developed HA-VTE and associated case fatality. (DOCX $24 \mathrm{~kb}$ )

Additional file 5: Annual incidence rates and adjusted rate ratios of patients who developed HA-VTE and associated case fatality. (DOCX 58 kb)

\section{Abbreviations}

AAA: Abdominal aortic aneurysm; APDC: Admitted patient data collection; CABG: Coronary-artery bypass graft; Cl: Confidence interval; DVT: Deep venous thrombosis; HA-VTE: Hospital-acquired venous thromboembolism; ICD-10-AM: International Statistical Classification of Diseases and Related Health Problems, Tenth Revision, Australian Modification; IR: Incidence rate; IRR: Incidence rate ratio; LOS: Length of stay; NSW: New South Wales; PE: Pulmonary embolism; SEIFA: Socio-economic indices for areas; VTE: Venous thromboembolism

\section{Acknowledgments}

Not applicable.

\section{Funding}

This work was partly supported by two National Health and Medical Research Council Project grants-Australia (APP1009916 and APP1020660). The funders had no role in study design, data collection and analysis, decision to publish, or preparation of the manuscript.

\section{Availability of data and materials}

The data that support the findings of this study are available from NSW APDC data custodian but restrictions apply to the availability of these data, which were used under license for the current study, and so are not publicly available. 


\section{Authors' contributions}

Conceived and designed the study: HA, JC. Prepared the data and performed the analyses: HA, JC, and LO. Wrote the paper: HA, JC, KH, and AF. All authors have read and approved the final version of the manuscript.

\section{Competing interests}

The authors declare that they have no competing interests.

\section{Consent for publication}

Not applicable.

\section{Ethics approval and consent to participate}

Ethical approval was obtained from the University of NSW Human Research Ethics Committee (LNR/11/CIPHS/64). Consent to participate was no applicable.

\section{Author details}

'Epidemiology and Health Analytics, Western Sydney Local Health Districts, Gungurra Building 68, Cumberland Hospital, 5 Fleet Street, North Parramatta 2151, NSW, Australia. ${ }^{2}$ Simpson Centre for Health Services Research-South Western Sydney Clinical School Faculty of Medicine, University of New South Wales, and Ingham Institute, Sydney, Australia. Intensive Care Unit, Royal Adelaide Hospital and School of Medicine, Faculty of Health Sciences, University of Adelaide, Adelaide, South Australia, Australia.

\section{Received: 4 September 2015 Accepted: 16 September 2016}

\section{Published online: 22 September 2016}

\section{References}

1. Geerts WH, Bergqvist D, Pineo GF, Heit JA, Samama CM, Lassen MR, et al. Prevention of venous thromboembolism: American College of Chest Physicians Evidence-Based Clinical Practice Guidelines. Chest. 2008;133(6 suppl):381S-453.

2. National Health and Medical Research Council. Preventing venous thromboembolism in hospitalised patients: summary of NHMRC activity 2003-2010. Melbourne: NHMRC; 2011

3. Hirsh J, Hoak J. Management of deep vein thrombosis and pulmonary embolism a statement for healthcare professionals from the Council on Thrombosis (in Consultation with the Council on Cardiovascular Radiology), American Heart Association. Circulation. 1996;93(12):2212-45.

4. Heit JA, Silverstein MD, Mohr DN, Petterson TM, O'Fallon WM, Melton III L. Risk factors for deep vein thrombosis and pulmonary embolism: a population-based case-control study. Arch Intern Med. 2000;160(6):809.

5. Heit JA, O'Fallon WM, Petterson TM, Lohse CM, Silverstein MD, Mohr DN, et at. Relative impact of risk factors for deep vein thrombosis and pulmonary embolism: a population-based study. Arch Intern Med. 2002:162(11):1245-8.

6. Lijfering WM, Rosendaal FR, Cannegieter SC. Risk factors for venous thrombosis-current understanding from an epidemiological point of view. Br J Haematol. 2010;149(6):824-33.

7. Kahn SR, Morrison DR, Emed J, Tagalakis V, Shrier I. Interventions for implementation of thromboprophylaxis in hospitalized medical and surgical patients at risk for venous thromboembolism. Cochrane Libr. 2010. doi:10.1002/14651858.CD008201.

8. Agnelli G. Prevention of venous thromboembolism in surgical patients. Circulation. 2004;110(24 suppl 1):IV-4-IV-12.

9. Piazza G, Fanikos J, Zayaruzny M, Goldhaber S. Venous thromboembolic events in hospitalised medical patients. Thromb Haemost. 2009;102(3):505-10

10. The Joint Commission. Performance measurement initiatives - national consensus standards for prevention and care of venous thromboembolism (VTE). 2007.

11. National Health and Medical Research Council. Clinical practice guideline for the prevention of venous thromboembolism (deep vein thrombosis and pulmonary embolism) in patients admitted to Australian hospitals. Melbourne: National Health and Medical Research Council; 2009.

12. Agency for Healthcare Research and Quality (AHRQ). Patient safety indicator v4 5 benchmark data tables. 2013.

13. Centers for Medicare and Medicaid Services. Hospital value-based purchasing (HVBP) program. Centers for Medicare and Medicaid Services 2013. http://www.cms.gov/Medicare/Quality-Initiatives-Patient-AssessmentInstruments/hospital-value-based-purchasing/index.html. Accessed 04 June 2015.

14. Reed K, May R. HealthGrades patient safety in American hospitals study. US. HealthGrades; 2011.
15. HealthGrades. Variation in patient safety outcomes and the importance of being informed. U.S: HealthGrades; 2013.

16. Semel ME, Lipsitz SR, Funk LM, Bader AM, Weiser TG, Gawande AA. Rates and patterns of death after surgery in the United States, 1996 and 2006 Surgery. 2012;151(2):171-82.

17. The Joint Commission. Improving America's hospitals. 2013.

18. Anderson Jr FA, Spencer FA. Risk factors for venous thromboembolism. Circulation. 2003:107(23 suppl 1):-I-9-I-16.

19. Naess IA, Christiansen SC, Romundstad P, Cannegieter SC, Rosendaal FR, Hammerstrøm J. Incidence and mortality of venous thrombosis: a population-based study. J Thromb Haemost. 2007;5(4):692-9.

20. Sliwka D, Fang MC. Venous thromboembolism prophylaxis in the United States: still room for improvement. J Gen Intern Med. 2010; 25(6):484-6.

21. Assareh H, Chen J, Ou L, Hollis SJ, Hillman K, Flabouris A. Rate of venous thromboembolism among surgical patients in Australian hospitals: a multicentre retrospective cohort study. BMJ Open. 2014 4(10):e005502.

22. Access Economics. The burden of venous thromboembolism in Australia. Report for the Australia and New Zealand working party on the management and prevention of venous thromboembolism. 2008.

23. Australian Institute of Health and Welfare (AIHW). VTE related hospitalisations in Australia: national hospital morbidity data 1999-2008. AlHW2010.

24. Ho WK, Hankey GJ, Eikelboom JW. The incidence of venous thromboembolism: a prospective, community-based study in Perth, Western Australia. Med J Aust. 2008;189(3):144-7.

25. National Centre for Classification in Health N. Australian Refined Diagnosis Related Groups (AR-DRGs), Version 6.0. Sydney: NCCH, Faculty of Health Sciences, The University of Sydney; 2010.

26. National Centre for Classification in Health N. Australian coding standards for ICD-10-AM and ACHI, Fifth Edition. Sydney: NCCH, Faculty of Health Sciences, The University of Sydney; 2006.

27. Lujic S, Watson DE, Randall DA, Simpson JM, Jorm LR. Variation in the recording of common health conditions in routine hospital data: study using linked survey and administrative data in New South Wales, Australia. BMJ Open. 2014;4(9):e005768. doi:10.1136/bmjopen-2014-005768.

28. Powell H, Lim LL, Heller RF. Accuracy of administrative data to assess comorbidity in patients with heart disease: an Australian perspective. J Clin Epidemiol. 2001;54(7):687-93.

29. Goldsbury DE, Armstrong K, Simonella L, Armstrong BK, O'Connell DL. Using administrative health data to describe colorectal and lung cancer care in New South Wales, Australia: a validation study. BMC Health Serv Res. 2012; 12(1):1-9. doi:10.1186/1472-6963-12-387.

30. Australian Institute of Health and Welfare (AlHW). Australian hospital statistics 2012-13. Health Services Series No. 54. Cat. No. HSE 145. Canberra: AlHW; 2014.

31. The Organisation for Economic Co-operation and Development (OECD). Definitions for health care quality indicators: 2012-2013 HCQI data collection. 2013.

32. Victorian Government Health Information. Patient safety indicatorstranslated technical specifications. Melbourne: Victorian State Government, Department of Health; 2006.

33. Agency for Healthcare Research and Quality. AHRQ quality indicators: patient safety indicators: technical specifications (Version 4.1), Agency for Healthcare Research and Quality. 2009.

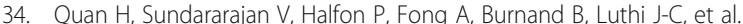
Coding algorithms for defining comorbidities in ICD-9-CM and ICD-10 administrative data. Med Care. 2005:43(11):1130-9.

35. Australian Bureau of Statistics. Census of population and housing: socioeconomic indexes for areas (SEIFA), Australia. Canberra: Australian Bureau of Statistics: 2011.

36. Demand and Performance Evaluation. NSW health services comparison data book 2008/2009. Sydney: NSW Ministry of Health; 2010.

37. Assareh H, Achat HM, Stubbs JM, Guevarra VM, Hill K. Incidence and variation of discrepancies in recording chronic conditions in Australian hospital administrative data. PLoS One. 2016;11(1):e0147087. doi:10.1371/journal.pone.0147087.

38. Welch HG, Sharp SM, Gottlieb DJ, Skinner JS, Wennberg JE. Geographic variation in diagnosis frequency and risk of death among Medicare beneficiaries. JAMA. 2011;305(11):1113-8. 
39. Assareh $H$, Achat HM, Guevarra VM, Stubbs JM. Effect of change in coding rules on recording diabetes in hospital administrative datasets. Int J Med Inform. 2016. doi:10.1016/j.jimedinf.2016.07.014.

40. R Core Team. R: A Language and Environment for Statistical Computing. 312th ed. Vienna: R Foundation Statistical Computing; 2013.

41. StataCorp. Stata Statistical Software: Release 11. College Station: StataCorp LP; 2009.

42. Khanna R, Maynard G, Sadeghi B, Hensley L, Medvedev S, Danielsen B, et al. Incidence of hospital-acquired venous thromboembolic codes in medical patients hospitalized in academic medical centers. J Hosp Med. 2014;9(4):221-5.

43. Rothberg MB, Lindenauer PK, Lahti M, Pekow PS, Selker HP. Risk factor model to predict venous thromboembolism in hospitalized medical patients. J Hosp Med. 2011;6(4):202-9.

44. Spyropoulos AC, Anderson JFA, FitzGerald G, Decousus H, Pini M, Chong BH, et al. Predictive and associative models to identify hospitalized medical patients at risk for vte. Chest. 2011:140(3):706-14. doi:10.1378/chest.10-1944.

45. White $\mathrm{RH}$. The epidemiology of venous thromboembolism. Circulation. 2003;107(23 suppl 1):I-4-I-8

46. White $\mathrm{RH}$, Zhou H, Romano PS. Incidence of symptomatic venous thromboembolism after different elective or urgent surgical procedures. Thromb Haemost. 2003;90(3):446-55.

47. National Institute of Clinical Studies (NICS). Evidence practice gaps report Volume 1. A review of developments 2004-2007. Melbourne: NICS; 2008.

48. Cohen AT, Tapson VF, Bergmann J-F, Goldhaber SZ, Kakkar AK, Deslandes B, et al. Venous thromboembolism risk and prophylaxis in the acute hospital care setting (ENDORSE study): a multinational cross-sectional study. Lancet. 2008;371(9610):387-94.

49. Phillips NM, Heazlewood VJ. Venous thromboembolism prophylaxis audit in two Queensland hospitals. Intern Med J. 2013;43(5):560-6.

50. Anderson Jr FA, Goldhaber SZ, Tapson VF, Bergmann J-F, Kakkar AK, Deslandes B, et al. Improving practices in US hospitals to prevent venous thromboembolism: lessons from ENDORSE. Am J Med. 2010;123(12):1099-106.e8.

51. Alikhan R, Peters F, Wilmott R, Cohen A. Fatal pulmonary embolism in hospitalised patients: a necropsy review. J Clin Pathol. 2004;57(12):1254-7.

52. Cohen A, Edmondson R, Phillips M, Ward V, Kakkar V. The changing pattern of venous thromboembolic disease. Pathophysiol Haemost Thromb. 1996; 26(2):65-71.

53. Hill J, Treasure T. GUIDELINES: reducing the risk of venous thromboembolism in patients admitted to hospital: summary of NICE guidance. BMJ. 2010;340(7740):259-60.

54. Barba R, Zapatero A, Losa JE, Marco J, Plaza S, Canora J, et al. Venous thromboembolism in acutely ill hospitalized medical patients. Thromb Res. 2010;126(4):276-9.

55. Ghaferi AA, Birkmeyer JD, Dimick JB. Hospital volume and failure to rescue with high-risk surgery. Med Care. 2011;49(12):1076-81.

56. Katz JN, Barrett J, Mahomed NN, Baron JA, Wright RJ, Losina E. Association between hospital and surgeon procedure volume and the outcomes of total knee replacement. J Bone Joint Surg. 2004;86(9):1909-16.

57. Ou L, Chen J, Assareh H, Hollis SJ, Hillman K, Flabouris A. Trends and variations in the rates of hospital complications, failure-to-rescue and 30Day mortality in surgical patients in New South Wales, Australia, 2002-2009. PLoS One. 2014:9(5):e96164.

58. Vartak S, Ward MM, Vaughn TE. Do postoperative complications vary by hospital teaching status? Med Care. 2008;46(1):25-32

59. Millar J, Mattke S. Members of the OECD patient safety panel. France: Selecting indicators for patient safety at the health systems level in OECD countries; 2004.

60. Chen J. Public reporting of health system performance: a rapid review of evidence on impact on patients, providers and healthcare organisations. Sydney: Sax Institute; 2010.

61. Jackson TJ, Michel JL, Roberts RF, Jorm CM, Wakefield JG. A classification of hospital-acquired diagnoses for use with routine hospital data. Med J Aust. 2009;191(10):544

62. National Institute of Clinical Studies. The incidence and risk factors for venous thromboembolism in hospitals in Western Australia 1999-2001, Prepared by the school of population health, University of Western Australia. Melbourne: NICS; 2005.
63. Parkin L, Sweetland S, Balkwill A, Green J, Reeves G, Beral V. Body mass index, surgery, and risk of venous thromboembolism in middle-aged women: a cohort study. Circulation. 2012;125(15):1897-904. doi:10.1161/circulationaha.111.063354.

64. Cheng Y-J, Liu Z-H, Yao F-J, Zeng W-T, Zheng D-D, Dong Y-G, et al. Current and former smoking and risk for venous thromboembolism: a systematic review and meta-analysis. PLoS Med. 2013;10(9):e1001515. doi:10.1371/journal.pmed.1001515.

\section{Submit your next manuscript to BioMed Central and we will help you at every step:}

- We accept pre-submission inquiries

- Our selector tool helps you to find the most relevant journal

- We provide round the clock customer support

- Convenient online submission

- Thorough peer review

- Inclusion in PubMed and all major indexing services

- Maximum visibility for your research

Submit your manuscript at www.biomedcentral.com/submit 